\title{
Coexistence of Idiopathic Parkinson's Disease and Creutzfeldt-Jakob Disease
}

\author{
C. Ezrin-Waters, L. Resch, A.E. Lang
}

\begin{abstract}
The association of idiopathic Parkinson's disease and Creutzfeldt-Jakob disease has not been reported previously. We describe the clinical findings in a patient with both disorders, confirmed at autopsy. The differential diagnosis of parkinsonism with cognitive and psychiatric disturbances is reviewed with emphasis on conditions which may complicate the course of idiopathic Parkinson's disease. The etiological implications of this combination are considered.
\end{abstract}

\begin{abstract}
RÉSUMÉ: Co-existence de la maladie de Parkinson idiopathique et de la maladie de Creutzfeldt-Jakob chez un patient L'association de la maladie de Parkinson idiopathique et de la maladie de Creutzfeldt-Jakob n'a pas été rapporté préalablement. Nous décrivons la présentation clinique chez un patient ayant les deux maladies, ainsi que la confirmation post-mortem. Nous passons en revue le diagnostic différentiel du parkinsonisme avec modifications cognitives et psychiatriques, mettant l'emphase sur les conditions compliquant l'évolution du Parkinson idiopathique. Nous considérons également les implications étiologiques de cette combinaison.
\end{abstract}

Can. J. Neurol. Sci. 1985; 12:272-273

The combination of parkinsonism with dementia or psychiatric changes is a common clinical problem. We report a patient presenting with parkinsonism who later developed psychiatric and cognitive changes. Postmortem examination revealed coexisting Creutzfeldt-Jakob (CJD) and idiopathic Parkinson's disease (IPD). The diagnostic and etiological considerations raised by this association are discussed.

\section{CASE RePORT}

A 68 year old female presented with slowly progressive akinesia, shuffling gait and resting tremor. All of these symptoms responded to Sinemet 100/25 q.i.d. and trihexyphenidyl $2 \mathrm{mg}$ t.i.d. She remained improved for $21 / 2$ years, when she developed confusion and nocturnal visual hallucinations. Worsening hallucinations, inability to read and paranoid psychosis prompted admission to another hospital. Initially it was thought that she was experiencing psychiatric complications from her antiparkinsonian medication. Drug withdrawal aggravated her parkinsonism but failed to improve the psychiatric disturbances. She was then transferred to the Toronto Western Hospital.

On examination she was disoriented and confused. Normal pupillary light response and funduscopic examination combined with attention to auditory but not visual stimuli, suggested the presence of cortical blindness. Her limbs were both rigid and spastic, the arms flexed at the elbows, hands held tightly closed and the legs held in extension. There was no resting tremor. Myoclonus, accentuated by startle, developed early in the hospital course. Reflexes were hyperactive and the right plantar response was extensor. There were bilateral grasp reflexes. The remainder of the general physical examination was normal.
Routine hematological, biochemical and CSF studies were normal. A CT-scan showed mild generalized atrophy. An EEG demonstrated periodic sharp-wave complexes at a rate of 1 per second. A clinical diagnosis of Creutzfeldt-Jakob disease was made. She deteriorated rapidly and died six months after the onset of her confusional symptoms.

Autopsy revealed a slightly atrophic brain with a thinned cortex. Histological examination revealed varying degrees of neuronal loss, spongiosis and gliosis throughout the cerebral and cerebellar cortices and lenticular nuclei (Figure 1). The substantia nigra and locus ceruleus showed neuronal loss, gliosis, loss of melanin pigment, and Lewy body inclusions in some remaining neurons (Figure 2).

\section{Discussion}

The association of parkinsonism with cognitive and psychiatric changes raises a number of diagnostic possibilities (Table 1). The course of IPD may be complicated by these changes for a number of reasons. It is well recognized that antiparkinsonian drugs can cause confusion, psychiatric and memory disturbances. Superimposed infection or metabolic disturbances frequently cause mental changes, particularly in elderly patients. Coexistent Alzheimer's disease may account for the dementia which occurs in some IPD patients ${ }^{1}$. Concomitant NPH and IPD rarely may explain parkinsonism combined with mental changes ${ }^{2}$. Furthermore, patients with Alzheimer's disease ${ }^{3}$ and $\mathrm{NPH}^{2,4}$ often have some degree of parkinsonism.

Parkinsonism is a well recognized manifestation of CreutzfeldtJakob disease ${ }^{5}$ but, to our knowledge, the combination of IPD 
and CJD has not been reported previously. This association in our patient presented considerable diagnostic difficulty. Initially it was thought that she demonstrated the typical psychiatric changes induced by antiparkinsonian drugs. When the diagnosis of CJD became clear, it was questioned whether this disorder had been the cause of her initial parkinsonian features. Although signs of parkinsonism have been early manifestations in some patients with CJD surviving longer than two years, usually these have been associated with other CNS features from the onset $^{6}$.

The etiology of IPD is unknown. Transmission studies using brain tissue from IPD patients have not resulted in neurological disease in animal recipients ${ }^{7}$. Pathological changes of IPD, notably the Lewy body, have not been present in brains of animals inoculated with transmissible CJD tissue. IPD is a common condition. These factors combined argue against a shared etiological basis for the two disorders seen here. Although we favour simple coincidence as an explanation for this combination of IPD and CJD in our patient, planned transmission studies using tissue from various brain regions including cortex and substantia nigra may provide alternative insights into this unusual association.

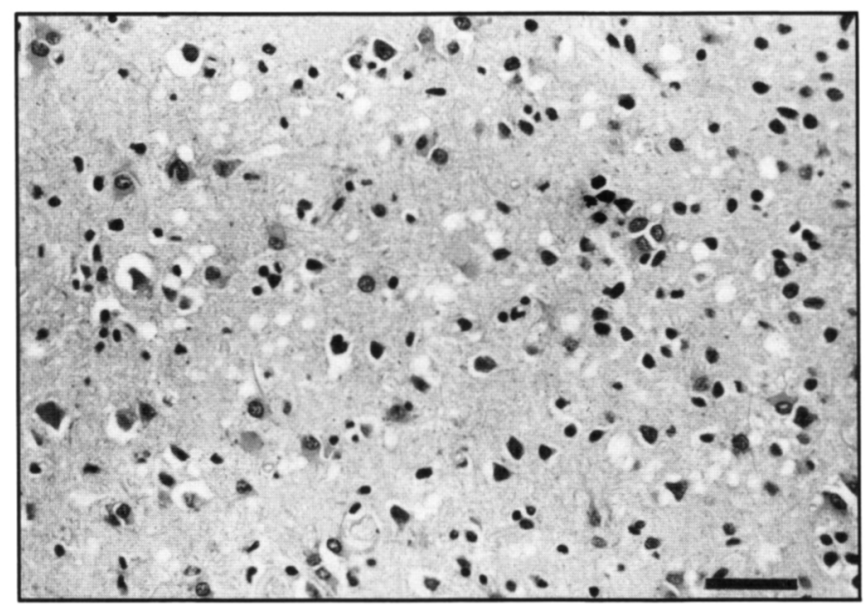

Figure I - Photomicrograph of temporal cortex showing neuronal loss astrogliosis, and spongiform change. Calibration bar equals $50 \mu \mathrm{m}$.

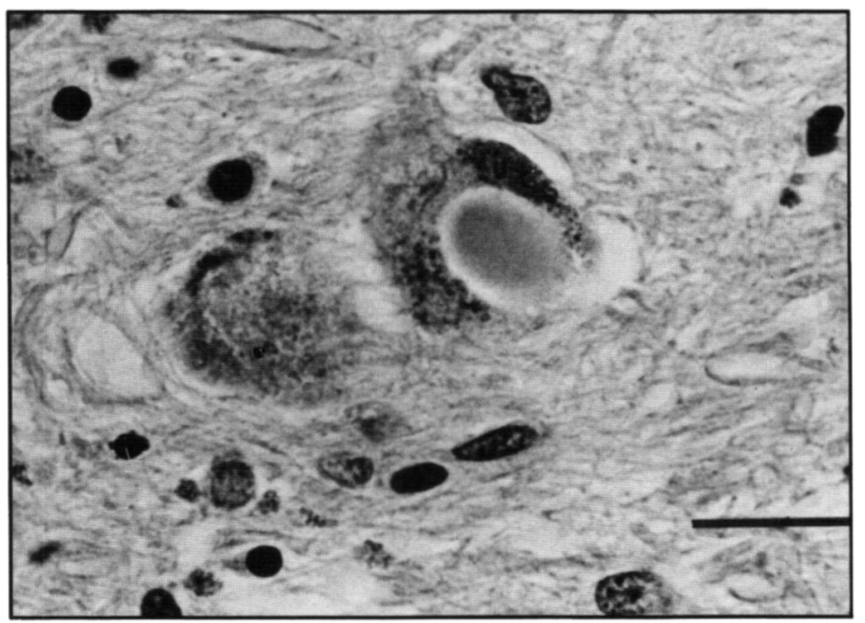

Figure 2 - Photomicrograph of substantia nigra showing astrogliosis and pigment in a macrophage. One of the neurons contains a large Lewy body. Calibration bar equals $25 \mu \mathrm{m}$.
Table 1: Diseases Causing Parkinsonism with Dementia and Psychiatric Changes

Idiopathic Parkinson's disease (with or without complicating factors) Alzheimer's disease

Creutzfeldt-Jakob disease

Pick's disease

Parkinson-Dementia - ALS Complex of Guam

Progressive Supranuclear Palsy

Multiple System Atrophy

Huntington's Chorea

Hallervorden-Spatz disease

Calcification of the Basal Ganglia

Wilson's disease

Other "Neurometabolic" diseases, e.g., Lafora Body Disease Ceroid Lipofuscinosis

Multi-infarct state ("atherosclerotic parkinsonism")

Multiple Head Injuries (Dementia Pugilistica)

Cerebral Anoxia (including $\mathrm{CO}$ poisoning)

Others (e.g., Manganese poisoning, neurosyphilis)

\section{ACKNOWLEDGEMENT}

Special thanks to Dr. P. Watson for referring this patient to us.

\section{REFERENCES}

1. Hakim AM, Mathieson G. Dementia in Parkinson's disease: A neuropathologic study. Neurology 1979; 29: 1209-1214.

2. Jacobs L, Conti D, Kinkel WR, et al. "Normal-pressure" hydrocephalus. Relationship of clinical and radiographic findings to improvement following shunt surgery. JAMA 1976; 235: 510-512.

3. Molsa PK, Marttila RJ, Rinne UK. Extrapyramidal signs in Alzheimer's disease. Neurology 1984; 34: 1114-1116.

4. Sypert CW, Leffman H, Ojemann GA. Occult normal pressure hydrocephalus manifested by Parkinsonism dementia complex. Neurology 1973; 23: 234-238.

5. Brown P, Cathala F, Sadowsky D, et al. Creutzfeldt-Jakob disease in France: Il. Clinical characteristics of 124 consecutive verified cases during the decade 1968-1977. Ann Neurol 1979; 6: 430-437.

6. Brown P, Rodgers-Johnson P, Cathala F et al. Creutzfeldt-Jakob disease of long duration: clinicopathological characteristics. transmissibility, and differential diagnosis. Ann Neurol 1984; 16: 295-304.

7. Gibbs CJJr, Gadjusek DC. Amyotrophic Lateral Sclerosis. Parkinson's disease and the amyotrophic lateral sclerosis-Parkinsonism-dementia complex of Guam: A review and summary of attempts to demonstrate infection as the etiology. J Clin Path 1972; 25 (suppl 6): 132-140. 